\title{
Rosa multiflora with Root Rot Tolerance Has No Tolerance Mechanism on the Root Surface during the Early Infection Process
}

\author{
Lianhua Li ${ }^{1}$, Koji Kageyama ${ }^{2}$, Wenjin $\mathbf{Y u}^{1}$ and Hirokazu Fukui ${ }^{3 *}$ \\ ${ }^{1}$ The United Graduate School of Agricultural Science, Gifu University, Yanagido, Gifu 501-1193, Japan \\ ${ }^{2}$ River Basin Research Center, Gifu University, Yanagido, Gifu 501-1193, Japan \\ ${ }^{3}$ Faculty of Applied Biological Science, Gifu University, Yanagido, Gifu 501-1193, Japan
}

\begin{abstract}
Rosa multiflora 'Matsushima No. 3' shows tolerance to root rot disease caused by Pythium helicoides Drechsler. However, there has been no report on the tolerance mechanism of 'Matsushima No. 3'. The infection process of Pythium in diseased plants was divided broadly into two stages, as early and later infection processes. This study focused on the early infection process: chemotaxis, attachment, and encystment of zoospores and germ tube germination on the root surface, in order to clarify the tolerance of 'Matsushima No. 3 ' in comparison with a susceptible rose. 'Matsushima No. 3' and $R$. 'Fashion Parade', which were estimated as showing tolerance and susceptibility to root rot disease in the field, respectively, were used as plant materials. Rooted cuttings of 'Matsushima No. 3' and 'Fashion Parade' were inoculated by zoospore suspension of $P$. helicoides, and root rot symptoms of these roses were evaluated at 7 days after inoculation. From the results of inoculation, we concluded that 'Matsushima No.3' was tolerant, and 'Fashion Parade' was susceptible to root rot disease. Visual observation showed that zoospores of $\boldsymbol{P}$. helicoides aggregated at the end of capillaries filled with root extract agar of 'Matsushima No. 3' as well as 'Fashion Parade', and zoospores encysted on the root surface of 'Matsushima No. 3' too. Therefore, 'Matsushima No. 3' didn't inhibit the chemotaxis and encystment of zoospores. From SEM observations, most cystospores germinated on the root surface in 'Matsushima No. 3' and 'Fashion Parade', and the percentages of germ tube penetration to the epidermis in 'Matsushima No. 3' and 'Fashion Parade' were 55.0 and 71.2, respectively. The result that no differences were recognized between the two roses during the early infection process indicated that the tolerance mechanism in 'Matsushima No. 3' was not active in the early but in the latter infection process, that is, some tolerance mechanism might inhibit hyphae from expanding to the cortical tissue.
\end{abstract}

Key Words: chemotaxis, encystment, Pythium helicoides, rose, tolerance mechanism.

\section{Introduction}

Root rot disease caused by Pythium helicoides Drechsler has brought heavy damage to cut rose production (Kageyama et al., 1998). It was estimated that Rosa multiflora 'Matsushima No. 3' showed tolerance against this disease in the field of cut rose production, and such tolerance in 'Matsushima No. 3' has been confirmed by bioassay in a previous paper ( $\mathrm{Li}$ et al., 2007). Although the phytopathological characteristics of $P$. helicoides were investigated (Kageyama et al., 2002, 2003), there was no report on the tolerance mechanism of 'Matsushima No. 3' to root

Received; August 10, 2006. Accepted; December 12, 2006.

* Corresponding author (E-mail: fukui@gifu-u.ac.jp). rot disease. Understanding the mechanism of hostpathogen interaction is very important for breeding and developing disease-tolerant rootstock.

The infection process of Pythium in diseased plants was reported by some papers, involving the guidance of zoospores to the root surface by chemo-attractants, attachment, and encystment of zoospores on the root surface, germ tube germination (early infection process), and penetration of the germ tube to the root epidermis, cortex, endodermis, and vascular stele (later infection process) (Deacon and Donaldson, 1993; Jones et al., 1991; Rey et al., 1998). The tolerance of 'Matsushima No. 3' to root rot disease might occur during some steps in these infection processes.

Concerning the resistance mechanism in the early infection process, we have reported that $R$. 'PEKcougel', which was resistant to crown gall disease, had less 
acetosyringone derivatives for chemo-attractants than low-resistant cultivars. Resistance also inhibited Agrobacterium tumefaciens, which causes root crown gall disease, from attaching to cell surfaces by mass secretions (Tan et al., 2005). On a resistance mechanism in a later process, Xi et al. (2000) reported that host cell wall alteration in barley prevented the germ tube of Rhynchosporium secalis from penetrating to epidermal cells, and Widmer et al. (1998) suggested that the tolerance mechanism of citrus against fibrous root rot disease reduced hyphal colonization in the cortex.

In a previous report ( $\mathrm{Li}$ et al., 2007), we observed that the density of hyphae in the cortical tissue of tolerant 'Matsushima No. 3' was lower than that in susceptible $R$. 'Nakashima 91'. The difference in hyphal density between the two roses might be due to the expression of tolerance in an early and/or later infection process. This study focused on the early infection process in order to clarify the interaction of 'Matsushima No. 3' and P. helicoides, and tolerant 'Matsushima No. 3' was investigated in comparison with a susceptible rose.

\section{Materials and Methods}

\section{Resistant test}

Rooted cuttings of 'Matsushima No. 3' and $R$. 'Fashion Parade' were used as plant materials. 'Matsushima No. 3' showed tolerance against P. helicoides, and 'Fashion Parade' was injured by root rot disease in potted rose production in Gifu Prefecture, Japan. Zoospores of $P$. helicoides B-5 were collected by the grass blades method (Kageyama et al., 2002; Waterhouse, 1967), and a zoospore suspension of $6.0 \times 10^{3}$ zoospores $/ \mathrm{mL}$ was used as the inoculation inoculum.

The inoculation method of zoospore suspension to rooted cuttings was the same as in a previous report (Li et al., 2007). Root rot was evaluated at 7 days after inoculation. Root symptoms were visually estimated using a disease severity scale from 0 to $3: 0=$ no root rot symptom was observed (healthy and white root), 0.5 $=\mathrm{a}$ part of the root was slightly brown, $1=\mathrm{a}$ part of the root was brown, $1.5=50 \%$ of the root was brown, $2=$ $75 \%$ of the root was brown, $2.5=$ the root was completely brown but the plant survived, and $3=$ the root was completely brown and the plant died.

Root rot severity $=\Sigma$ (disease index $\times$ number of plants at each severity)/(maximum disease index

$\times$ total number of plants) $\times 100$

The experiment was repeated three times.

\section{Optical microscope examination}

In optical microscope and Scanning Electron Microscope (SEM) examinations, the sterile roots differentiated and elongated in vitro were used with the purpose of preventing other fungi from disturbing accurate observations. Although we reported that 'Nakashima 91' was susceptible in a previous paper
(Li et al., 2007), shoot tips of 'Nakashima 91' couldn't be proliferated in vitro in spite of culturing on various media. So, the chemotaxis and encystment of zoospores and germ tube germination on the root surface in 'Matsushima No. 3' were compared with those of 'Fashion Parade' instead of 'Nakashima 91' in this study.

Shoot tips of 'Matsushima No. 3' and 'Fashion Parade' were cultured at $25^{\circ} \mathrm{C}$ under $3000 \mathrm{~lx}$ with a $16 \mathrm{~h}$ photoperiod on MS medium (Murashige and Skoog, 1962) containing 3\% sucrose and $0.2 \%$ gelrite supplemented with $10^{-6} \mathrm{M}$ 6-benzylaminopurine (BAP) and $10^{-7} \mathrm{M}$ gibberellin $\mathrm{A} 3\left(\mathrm{GA}_{3}\right)$ or $10^{-5} \mathrm{M}$ BAP and $10^{-6} \mathrm{M} \mathrm{GA}_{3}$, respectively. Shoot nodes were cut out from elongated shoots after six weeks and were subcultured every six weeks. After subculture, the base of elongated shoots was dipped into $10^{-3} \mathrm{M} 3$-indolebutyric acid (IBA) for $30 \mathrm{~min}$, and then shoots were cultured on MS medium containing $3 \%$ sucrose and $0.2 \%$ gelrite without hormones. The differentiated and elongated roots were cut from the shoot and washed carefully in distilled water to remove agar medium several times for use as experimental materials.

\section{1) Chemotaxis of zoospores}

Root extract was obtained by grinding roots from 3 test-tube cuttings and it was mixed with $15 \mathrm{~mL}$ of $1.6 \%$ agar medium. After heating medium at $40^{\circ} \mathrm{C}$, the capillary tube was filled with agar medium with root extract and it was put on a slide glass and covered with a cover glass. A zoospore suspension of $3.0 \times 10^{3}$ zoospores $/ \mathrm{mL}$ was dropped around the capillary tube (Royle and Hickman, 1964). After inoculation for $30 \mathrm{~min}$, zoospores were stained with aceto-carmine and observed under an optical microscope. Agar medium without root extract was tested as a control.

\section{2) Attachment of zoospores on the root surface}

For optical microscope observation, the root was cut off and put on a slide glass. A zoospore suspension of $3.0 \times 10^{3}$ zoospores $/ \mathrm{mL}$ was dropped on the root, and then a cover glass was put on the root. Thirty min after inoculation, cystospores on the root surface were counted every $2 \mathrm{~mm}$ from the root tip. Four experimental repetitions were performed with three roots.

For SEM observation, roots were soaked in $1.0 \times 10^{3}$ zoospores $/ \mathrm{mL}$ zoospore suspension for $2 \mathrm{~h}$ at $30^{\circ} \mathrm{C}$ in the darkness, and then $5 \mathrm{~mm}$ root segments from the root tips were excised from the roots of 'Matsushima No. 3' and 'Fashion Parade', respectively. Ten segments from each of the two roses were fixed for $2 \mathrm{~h}$ with $2.5 \%$ glutaraldehyde and $2 \%$ paraformaldehyde in $50 \mathrm{mM}$ phosphate buffer $(\mathrm{PB}, \mathrm{pH}=7.2)$ at room temperature, and then washed with $25 \mathrm{mM}$ PB for $20 \mathrm{~min} 3$ times. Segments were post-fixed for $1 \mathrm{~h}$ with $2 \%$ osmic acid in $50 \mathrm{mM} \mathrm{PB}$, and then they were washed with $25 \mathrm{mM}$ PB once. Subsequently, the segments were dehydrated with ethanol and substituted to isoamyl acetate. At last, critical-point drying and platinum palladium coating in an ion sputter were carried out, and cystospores on the 
root surface were observed by SEM (S-4300; Hitachi, Ltd., Japan).

\section{Results and Discussion}

Figure 1 shows the percentages of each disease index at 7 days after inoculation in 'Matsushima No. 3' and 'Fashion Parade' in this inoculation, and the results of 'Matsushima No. 3' and 'Nakashima 91' in a previous paper (Li et al., 2007) were written concurrently too. The root rot severity of 'Matsushima No. 3' in this study and the previous paper was 10.5 and 4.8 , respectively. The severity in this study was slightly higher than in the previous report, but the percentage with a disease severity scale of 0 : healthy and white root, in this study was higher than that in the previous paper, and the difference between the two disease severities only depended on $2.6 \%$ with a disease severity scale of 3.0 in this study. Although the root rot severity of 'Matsushima No. 3' in this study was slightly higher than that in the previous paper, the percentage of healthy and white roots was higher in this study than in the previous paper, and the root rot severity of 'Matsushima No. 3' in this study was obviously lower than 25.8 in susceptible 'Nakashima 91'. Therefore, we concluded that 'Matsushima No. 3' was tolerant to root rot disease.

'Matsushima No. 3' showed not true resistance but

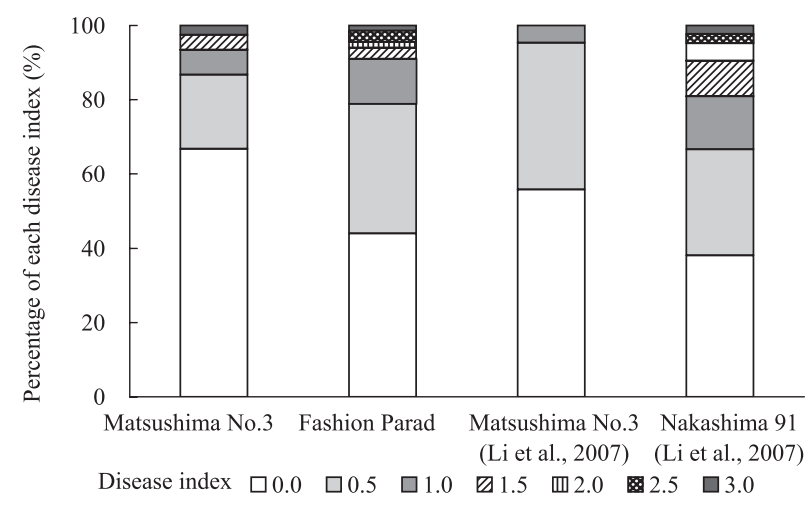

Fig. 1. Percentage of each disease index at 7 days after inoculation with Pythium helicoides in 'Matsushima No. 3' and 'Fashion Parade' in this inoculation and 'Matsushima No. 3' and 'Nakashima 91' in a previous paper (Li et al., 2007). field resistance (Li et al., 2007). Environmental and plant conditions often influence field resistance. Although the conditions of cutting and the inoculation method in this study were the same as in the previous study, cutting was carried out in different seasons: the previous experiment was carried out in spring and this experiment was conducted in autumn. In general, plants have a stronger growth ability in spring than in other seasons, and rooted cuttings in spring might have a higher tolerance than in other seasons.

'Fashion Parade' was injured by root rot disease in potted rose production in Gifu Prefecture, and we have concluded that 'Fashion Parade' is susceptible. On comparing 'Fashion Parade' with susceptible 'Nakashima 91' (Fig. 1), although 'Fashion Parade' had a slightly higher percentage of healthy and white roots than 'Nakashima 91', the percentage of 0.5 in 'Fashion Parade' was higher and many roots with upper disease severity scales were observed also. Root rot severity in 'Fashion Parade' was significantly higher than that in 'Matsushima No. 3'; we confirmed, therefore, that 'Fashion Parade' is susceptible compared with 'Matsushima No. 3'.

\section{Guidance of zoospores by chemo-attractants and encystment and adhesion on the root surface}

No zoospore was observed at the end of capillaries filled with water agar (Fig. 2A). However, zoospores of $P$. helicoides after $30 \mathrm{~min}$ of inoculation aggregated at the end of capillaries filled with root extract agar of 'Matsushima No. 3' and 'Fashion Parade' (Fig. 2B, C). These results indicated that root extract of 'Matsushima No. 3' and 'Fashion Parade' had chemo-attractants for chemotaxis; nevertheless, 'Matsushima No. 3' was tolerant and 'Fashion Parade' was susceptible.

Acetosyringone derivatives were chemo-attractants of Agrobacterium tumefaciens. 'PEKcougel' and $R$. 'Lifirane', which were resistant roses against crown gall disease, secreted less acetosyringone derivatives than the susceptible roses, and one of the resistance mechanisms to crown gall disease was related to acetosyringone derivative exudation (Tan et al., 2004). In Pythium, amino acids, polysaccharides, or volatile
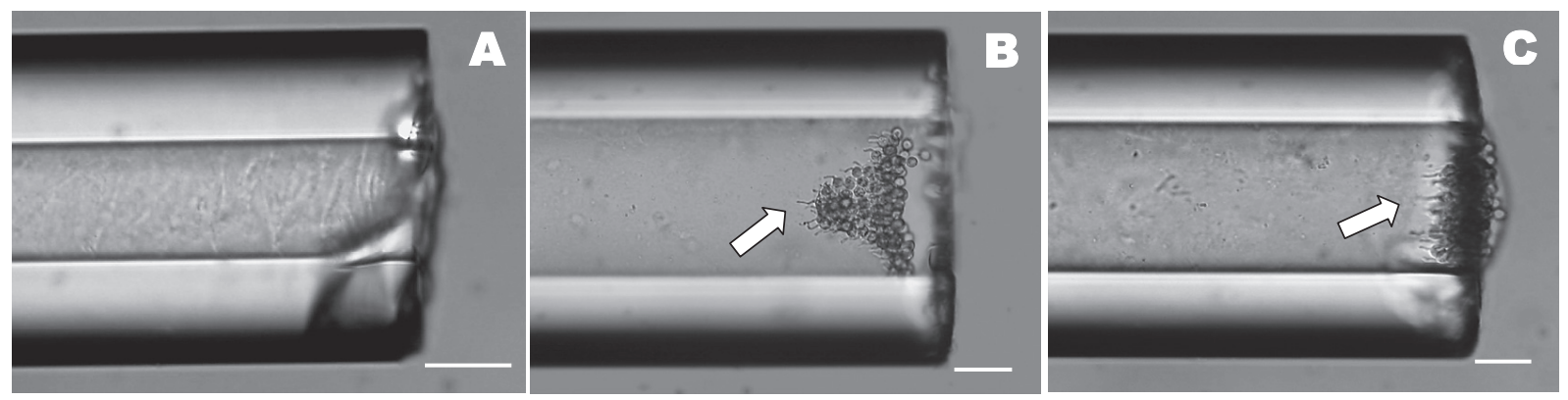

Fig. 2. Aggregation of zoospores of Pythium helicoides at the capillary end filled with root extract medium. A: Water agar medium, B: 'Matsushima No. 3' root extract medium, C: 'Fashion Parade' root extract medium. Arrows: Zoospores aggregated, Bars: $50 \mu \mathrm{m}$. 
compounds were demonstrated to stimulate chemotaxis of zoospores (Donaldson and Deacon, 1993; Jones et al., 1991). Although we had not identified the component of root extracts from 'Matsushima No. 3', 'Matsushima No. 3' did not lack the ability to biosynthesize these chemo-attractants. Dukes and Apple (1961) reported that zoospores of Phytophthora parassitica were attracted to tobacco roots both resistant and susceptible to blank shank disease. The zoospores of $P$. helicoides were attracted to both tolerant and susceptible roses in this study too. The tolerance mechanism in 'Matsushima No. 3', therefore, had no relation with chemotaxis.

Encystment of zoospores was observed after reaching the root surface (Fig. 3), and the number of cystospores on the root surface was counted using an optical microscope (Table 1). The number of cystospores of 'Matsushima No. 3' tended to be more than in 'Fashion Parade' at several distances from the root tip, but there was no significant difference between the two roses. On the other hand, a large number of cystospores in 'Matsushima No. 3' was present 0-2 $\mathrm{mm}$ from the root tip, and zoospores tended to encyst more at the root tip than at the base of the root. In 'Fashion Parade', the number of cystospores at $0-2 \mathrm{~mm}$ from the root tip was also significantly greater than those at $2-4,4-6$, and 8 $10 \mathrm{~mm}$. The encystment of zoospores was important for later infection; that is to say, attachment at a favorable location was effective for the germ tube to penetrate to the root surface. The observation of more cystospores at $0-2 \mathrm{~mm}$ from the root tip in 'Matsushima No. 3' and

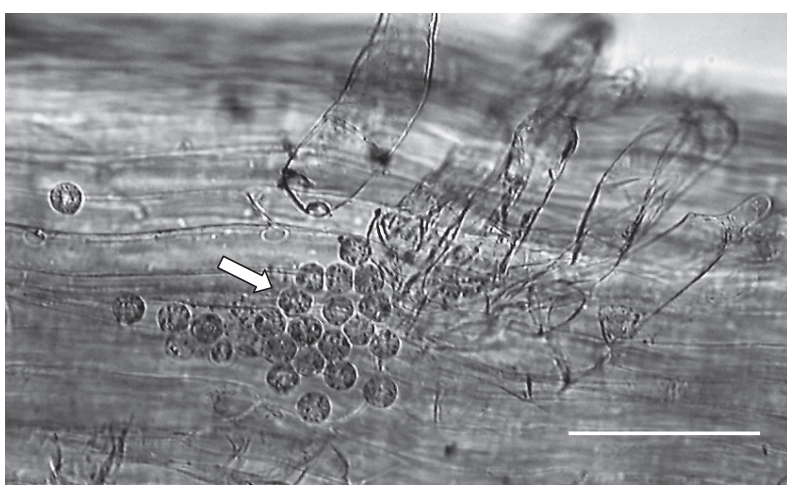

Fig. 3. Encystment of zoospores on the root surface in 'Fashion Parade' at $30 \mathrm{~min}$ after inoculation. Arrow: Cystospores, Bar: $50 \mu \mathrm{m}$.
'Fashion Parade' might have a relation to the underdevelopment of the cell wall of the epidermis. Some papers indicated that zoospores were attracted to the most susceptible sites on roots by chemotaxis (Donaldson and Deacon, 1993; Jones et al., 1991; Zentmyer, 1961). Thus, the root tip might be the most susceptible site.

At any rate, the observation of more cystospores on the root surface in 'Matsushima No. 3' did not support an inhibition mechanism in 'Matsushima No. 3' functioning at the stage of encystment in the infection process. This result was consistent with Goode's (1956) report that there was no significant difference in the encystment of Phytophthara fragariae zoospores on root surfaces of resistant and susceptible strawberry plants.

\section{Germ tube germination on the root surface}

The germination of cystospores was observed on the root surface at $2 \mathrm{~h}$ after inoculation in 'Matsushima No. 3' as well as 'Fashion Parade' by SEM (Fig. 4A), and most cystospores attached on the root surface germinated successfully (Table 2). Some cystospores elongated their germ tube along the root surface (Fig. 4B) and the average germ tube length of 'Fashion Parade' tended to be longer than in 'Matsushima No. 3' (Table 2). Direct penetration to the epidermis of the root (Fig. 4C) or an appressorium-like swelling in the germ tube (Fig. 4D) was observed, and the percentages of penetration in 'Fashion Parade' and 'Matsushima No. 3' were 71.2 and 55.0, respectively. Although the percentages of germination and penetration in 'Matsushima No. 3' were lower than those in 'Fashion Parade', we could not decide whether 'Matsushima No. 3 ' showed tolerance at the germination and penetration stages, which are important steps in the pathogenic processes (Warwar and Dickman, 1996), because these percentages in 'Matsushima No. 3' were high enough to infect the root.

An appressorium-like swelling was observed at the tip of the germ tube (Fig. 4D), but the percentage was very low in both 'Matsushima No. 3' and 'Fashion Parade'. Hardham (2001) reported that an appressorium was formed when it was difficult for germ tubes to penetrate into the epidermis. As most of the germ tubes in tolerant 'Matsushima No. 3' penetrated successfully into the epidermis without appressorium-like swellings,

Table 1. The number of cystospores at different root surfaces of Rosa multiflora 'Matsushima No. 3' and R. 'Fashion Parade' after 30 min inoculation with Pythium helicoides.

\begin{tabular}{lccccccccc}
\hline \hline & \multicolumn{7}{c}{ Distance from root tip (mm) } \\
\cline { 2 - 9 } & $0-2$ & $2-4$ & $4-6$ & $6-8$ & $8-10$ & $10-12$ & $12-14$ & $14-16$ \\
\hline Matsushima No. 3 & $18 \mathrm{a}^{\mathrm{z}}$ & $11 \mathrm{ab}$ & $10 \mathrm{~b}$ & $10 \mathrm{ab}$ & $13 \mathrm{ab}$ & $6 \mathrm{~b}$ & $9 \mathrm{~b}$ & $12 \mathrm{ab}$ \\
Fashion Parade & $16 \mathrm{a}$ & $6 \mathrm{~b}$ & $6 \mathrm{~b}$ & $12 \mathrm{ab}$ & $6 \mathrm{~b}$ & $8 \mathrm{ab}$ & $10 \mathrm{ab}$ & $7 \mathrm{ab}$ \\
\hline F-test & NS $^{\mathrm{y}}$ & NS & NS & NS & NS & NS & NS & NS \\
\hline
\end{tabular}

${ }^{\mathrm{z}}$ Different letters in the table indicate significant differences, $5 \%$ level $(\mathrm{n}=4)$, within cultivars.

y Each section of $2 \mathrm{~mm}$ for two cultivars shows no significant difference from each other $(\mathrm{n}=4)$. 

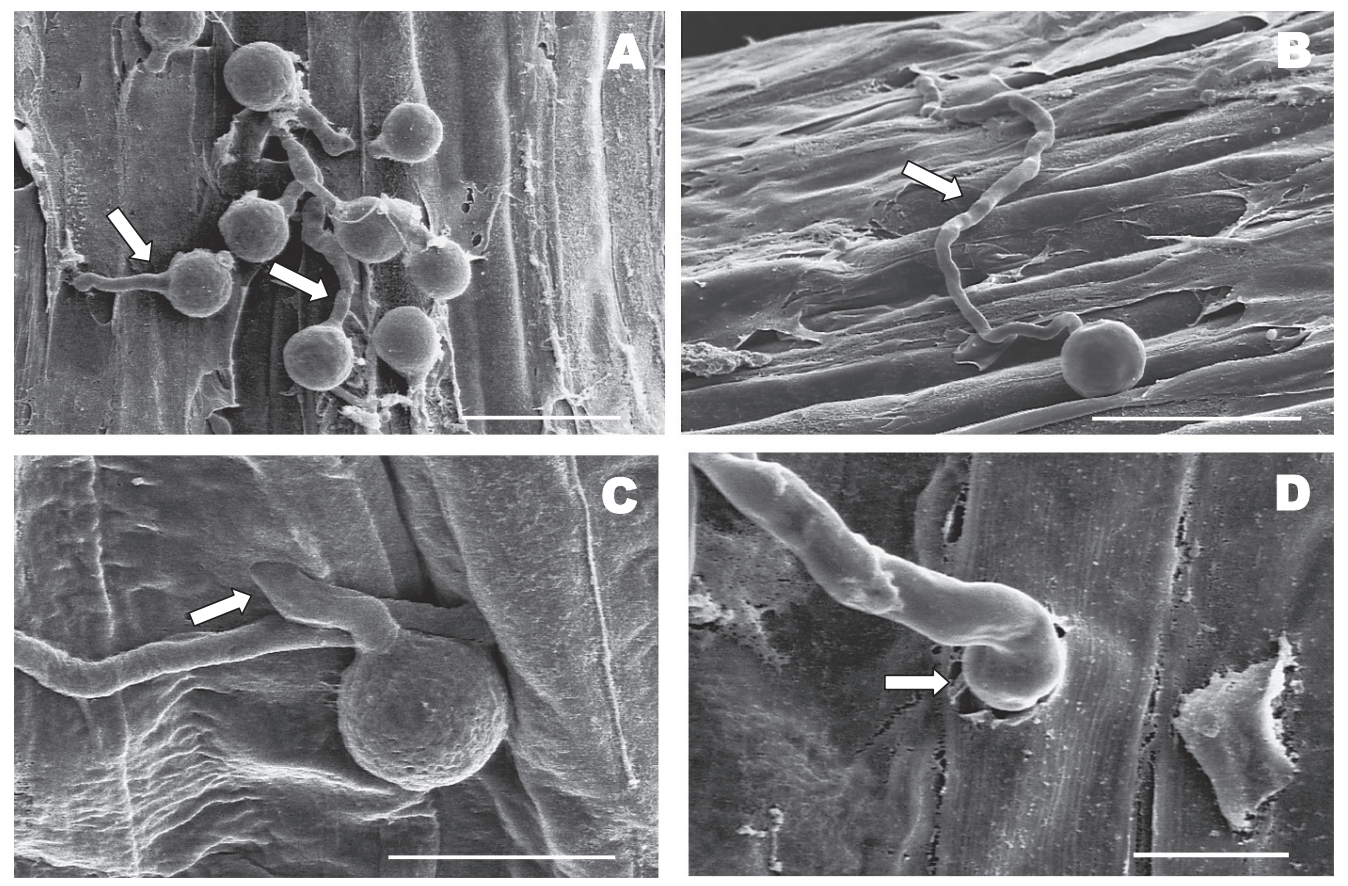

Fig. 4. Germination of cystospores (arrows) (A), elongation of the germ tube on the root surface (arrow) (B), direct penetration of the germ tube to the epidermis (arrow) (C), and appressorium-like swelling in the germ tube (arrow) (D). Bars: $25 \mu \mathrm{m}$ (A); $20 \mu \mathrm{m}$ (B); $10 \mu \mathrm{m}$ (C); $5 \mu \mathrm{m}$ (D).

Table 2. Germination, average germ tube length, formation of appressorium-like swelling, and penetration to the epidermis at $2 \mathrm{~h}$ after inoculation in Rosa multiflora 'Matsushima No. 3' and R. 'Fashion Parade'.

\begin{tabular}{lcccc}
\hline \hline & Germination $(\%)$ & $\begin{array}{c}\text { Average germ tube length } \\
(\mu \mathrm{m})\end{array}$ & $\begin{array}{c}\text { Formation of } \\
\text { appressorium-like } \\
\text { swelling }(\%)\end{array}$ & Penetration to epidermis $(\%)$ \\
\hline Matsushima No. 3 & 89.6 & 22.9 & 5.0 & 55.0 \\
Fashion Parade & 93.0 & 39.8 & 9.5 & 71.2 \\
\hline
\end{tabular}

as shown in Table 2, this formation did not necessarily have any relation with easy penetration to the epidermis of the root.

The infection process of Pythium in diseased plants was divided broadly into two stages. One involved the interaction of the plant and pathogen which took place outside the root tissue, and it was the early stage in the infection process. The other was the interaction which happened inside the root tissue, constituting the latter stage of the infection process. Up till now, we have reported that the density of hyphae in the cortical tissue of 'Matsushima No. 3' was lower than that of susceptible 'Nakashima 91' (Li et al., 2007). A lower density of hyphae in 'Matsushima No. 3' might be related to the expression of tolerance in the early and/or later infection process. In the early infection process, several tolerance mechanisms were involved, and these were as follows: less chemo-attractions, lower encystment and germination rates on the epidermis, and a lower penetration rate of the germ tube to the epidermis. From the results of this study, no difference was observed between 'Matsushima No. 3' and 'Fashion Parade' in terms of chemo-attractions, the encystment rate, germination rate, and percentage of germ tube penetration.

From these and the previous results, we could conclude that the tolerance mechanism in 'Matsushima No. 3' was not involved in the early but in the latter infection process, that is, 'Matsushima No. 3 ' might have some tolerance mechanism that inhibits hyphae from expanding to cortical tissue. Goode (1956) reported that strawberry resistant to red core disease caused by Phytophthora fragariae had no inhibitory mechanism involving attachment, encystment, and germination, and inhibited the growth of internal hyphae in cortical tissue after penetration of the germ tube. Our results were consistent with Goode's. We observed in preliminary research that roots of 'Matsushima No. 3' had antifungal substances to $P$. helicoides and the concentrations were higher than in 'Nakashima 91' (Nagaoka et al., 2006), and these antifungal substances might have a relation to the lower expansion of hyphae in cortical tissue of 'Matsushima No. 3'.

\section{Literature Cited}

Deacon, J. W. and S. P. Donaldson. 1993. Molecular recognition in the homing responses of zoosporic fungi, with special 
reference to Pythium and Phytophthora. Mycol. Res. 97: $1153-1171$.

Donaldson, S. P. and J. W. Deacon. 1993. Effect of amino acids and sugars on zoospore taxis, encystment and cyst germination in Pythium aphanidermatum (Edson) Fitzp., P. catenulatum Matthews and P. dissotocum Drechs. New Phytol. 123: 289-295.

Dukes, P. D. and J. L. Apple. 1961. Chemotaxis of zoospores of Phytophthora parasitica var. nicottianae by plant roots and certain chemical solutions. Phytopathol. Notes 195-197.

Goode, P. M. 1956. Infection of strawberry roots by zoospores of Phytophthora fragariae. Trans. Brit. Mycol. Soc. 39: 367377.

Hardham, A. R. 2001. The cell biology behind Phytophthora pathogenicity. Aust. Plant Pathol. 30: 91-98.

Jones, S. W., S. P. Donaldson and J. W. Deacon. 1991. Behavior of zoospore and zoospore cysts in relation to root infection by Pythium aphanidermatum. New Phytol. 117: 289-301.

Kageyama, K., H. Fukui, T. Aoyagi, T. P. Mcgonigle and Y. Matsubara. 1998. Root rot of rose caused by Pythium helicoides. Ann. Phytopathol. Soc. Jpn. 64: 629 (in Japanese).

Kageyama, K., T. Aoyagi, H. Sunouchi and H. Fukui. 2002. Root rot of miniature roses caused by Pythium helicoides. J. Gen. Plant Pathol. 68: 15-20.

Kageyama, K., M. Suzuki, A. Priyatmojo, Y. Oto, K. Ishiguro, H. Suga, T. Aoyagi and H. Fukui. 2003. Characterization and identification of asexual strains of Pythium associated with root rot of rose in Japan. J. Phytopathol. 151: 485-491.

Li, L., K. Kageyama, N. Kinoshita, W. Yu and H. Fukui. 2007. Development of bioassay for resistance to rose root rot disease caused by Pythium helicoides. J. Japan. Soc. Hort. Sci. 76: 79-84.

Murashige, T. and F. Skoog. 1962. A revised medium for rapid growth and bioassays with tobacco tissue cultures. Physiol. Plant. 15: 473-497.
Nagaoka, F., L. Li, K. Kageyama and H. Fukui. 2006. Antifungal substances to pythium root rot disease in rose root. J. Japan. Soc. Hort. Sci. 75 (Supple. 2): 352 (In Japanese).

Rey, P., B. Nicole and T. Yves. 1998. Ultrastrural and cytochemical investigation of asymptomatic infection by Pythium spp. Phytopathology 88: 234-244.

Royle, D. J. and C. J. Hickman. 1964. Analysis of factors governing in vitro accumulation of zoospores of Pythium aphanidermatum on roots. Canad. J. Microbiol. 10: 151-162.

Tan, B. S., K. Hasuo, S. Kawai, K. Kageyama, S. Matsumoto and H. Fukui. 2004. Resistance of roses to crown gall disease are related to the quantity of acetosyringone derivatives exudates. Acta Hort. 630: 207-212.

Tan, B. S., Y. Ishiguro, K. Kageyama, S. Matsumoto and H. Fukui. 2005. Blocking of attachment to cell wall and immobilization of bacteria as a resistance response to crown gall disease in roses. J. Japan. Soc. Hort. Sci. 74: 324-329.

Warwar, V. and M. B. Dickman. 1996. Effects of calcium and calmodulin on spore germination and appressorium development in Colletotrichum trifolii. Appl. Environ. Microbiol. 62: 74-79.

Waterhouse, G. E. 1967. Key to Pythium Pringsheim. Commonwealth Mycological Institute, Mycological Papers 109: 1-15.

Widmer, T. L, J. H. Graham and D. J. Mitchell. 1998. Histological comparison of fibrous root infection of disease-tolerant and susceptible Citrus hosts by Phytophthora nicotianae and P. palmivora. Phytopathology 88: 389-395.

Xi, K., P. A. Burnett, J. P. Tewari, M. H. Chen, T. K. Turkingtton and J. H. Helm. 2000. Histopathological study of barley cultivars resistant and susceptible to Rhynchosporium secalis. Phytopathology 90: 94-102.

Zentmyer, G. A. 1961. Chemotaxis of zoospores for root exudates. Science 133: 1595-1596. 